\title{
Monocyte chemoattractant protein-1 deficiency does not affect steatosis or inflammation in livers of mice fed a methionine-choline-deficient diet
}

\author{
Karen M Kassel ${ }^{1}$, Grace L Guo ${ }^{1}$, Ossama Tawfik ${ }^{2}$ and James P Luyendyk ${ }^{1}$
}

\begin{abstract}
Monocyte chemoattractant protein-1 (MCP-1, Ccl2) expression is increased in livers of patients with nonalcoholic steatohepatitis and in murine models of steatohepatitis. Several studies in rodents indicate that MCP-1 contributes to liver steatosis induced by feeding a high-fat diet. However, the extent of MCP-1 involvement in the widely utilized methionine-choline-deficient (MCD) diet model of steatohepatitis has not been determined. We tested the hypothesis that MCP-1 contributes to steatohepatitis in mice fed the MCD diet. MCP-1-deficient mice on a C57BI/6J background and age-matched C57BI/6J mice were fed either MCD diet or control diet for 4 weeks. MCP-1 deficiency did not affect steatohepatitis, as indicated by liver histopathology, nor did it affect serum alanine aminotransferase activity, hepatic triglyceride levels, hepatic inflammatory gene induction, or macrophage accumulation in mice fed the MCD diet. MCP-1 deficiency reduced the expression of the profibrogenic genes, pro-collagen 1a1, connective tissue growth factor, and transforming growth factor- $\beta$, in mice fed the MCD diet. MCP-1 deficiency significantly reduced collagen deposition and $\alpha$-smooth muscle actin protein levels in the livers of mice fed the MCD diet. The results indicate that MCP-1 does not contribute to liver steatosis or inflammation in the MCD diet model of steatohepatitis. Rather, the data suggest that MCP-1 contributes to fibrosis in mice fed the MCD diet, independent of effects on steatosis and inflammation. Laboratory Investigation (2010) 90, 1794-1804; doi:10.1038/labinvest.2010.143; published online 9 August 2010
\end{abstract}

KEYWORDS: fibrosis; inflammation; liver; MCP-1; steatohepatitis

Accumulation of fat within the liver (ie, steatosis) is commonly associated with diseases such as nonalcoholic fatty liver disease (NAFLD), the hepatic manifestation of metabolic syndrome. Although the mechanism is not entirely clear, steatosis can lead to nonalcoholic steatohepatitis (NASH), with evidence of both hepatocellular injury and chronic inflammation; this inflammation is generally considered a critical mediator of liver fibrosis in a subset of patients. Steatosis can be induced experimentally in rodents by altering the diet or by genetic approaches. The mechanisms whereby different experimental models induce steatosis vary, and this has been reviewed elsewhere. ${ }^{1}$ In contrast to models utilizing diets high in saturated fat (ie, $>20 \%$ of calories from fat), so-called Western diets, ${ }^{2-4}$ the widely utilized lipogenic methionine-choline-deficient (MCD) diet is not high in fat. Rather, the MCD diet causes defects in the handling of triglycerides, both at the level of transport and storage, as well as in de novo synthesis; these defects, combined with a high level of sucrose, lead to a rapid onset of steatohepatitis. ${ }^{5}$ Diets high in fat cause obesity and insulin resistance, whereas mice fed the MCD diet do not exhibit these characteristics. ${ }^{6}$ Given sufficient time, both diets cause steatohepatitis, although the mechanisms whereby fat accumulates in the liver in these two models are quite different.

The progression of fatty liver disease is associated with the expression of proinflammatory factors, including the cytokine monocyte chemoattractant protein-1 (MCP-1, Ccl2). MCP-1 mRNA is markedly increased in the livers of patients with steatosis and NASH. ${ }^{7-10}$ Serum levels of MCP-1 are elevated in patients with NASH compared with healthy individuals. $^{7}$ Similarly, hepatic MCP-1 mRNA and plasma protein levels are increased in mice fed a high-fat diet, ${ }^{2-4}$ and hepatic MCP-1 mRNA and MCP-1 protein levels in the plasma are increased in mice fed the MCD diet. ${ }^{11}$

\footnotetext{
${ }^{1}$ Department of Pharmacology, Toxicology and Therapeutics, The University of Kansas Medical Center, Kansas City, KS, USA and ${ }^{2}$ Department of Pathology and Laboratory Medicine, The University of Kansas Medical Center, Kansas City, KS, USA

Correspondence: Dr JP Luyendyk, PhD, Department of Pharmacology, Toxicology and Therapeutics, The University of Kansas Medical Center, 3901 Rainbow Boulevard, MS-1018, Kansas City, KS 66160, USA. E-mail: jluyendyk@kumc.edu
} 
MCP-1 could contribute to the pathogenesis of NASH through multiple mechanisms, and this could depend on the context in which steatosis occurs. Indeed, several recent studies indicate that MCP-1 contributes directly to hepatic lipid accumulation and insulin resistance in mice fed a highfat diet. For example, MCP-1 deficiency reduced hepatic triglyceride levels in mice fed a high-fat diet. $^{2}$ Moreover, a pharmacological antagonist of chemokine (C-C motif) receptor 2 (CCR2), the primary receptor for MCP-1, reduced hepatic steatosis in genetically obese diabetic $\mathrm{db} / \mathrm{db}$ mice. ${ }^{12}$ These studies suggest that MCP-1 contributes to steatosis induced by a high-fat diet.

Another mechanism whereby MCP-1 could contribute to the pathogenesis of NAFLD and NASH is by enhancing the inflammatory response. MCP-1 coordinates leukocyte recruitment to the liver by activation of the CCR2 receptor on inflammatory cells including monocytes/macrophages. ${ }^{13}$ Indeed, deficiency in either MCP-1 or CCR2 reduced hepatic infiltration of inflammatory cells in various models of liver disease, ${ }^{14-17}$ suggesting that MCP-1 could contribute not only to steatosis but also to inflammation. In addition, several recent studies indicate that MCP-1 is an important mediator of liver fibrosis. ${ }^{15,18,19}$ Together, these studies indicate that the effects of MCP-1 in NASH could be multifaceted and model dependent.

Although MCP-1 is induced in both high-fat and MCD diet models of NAFLD/NASH, fundamental differences in the mechanism of steatosis in each model exist. We sought to characterize the contribution of MCP-1 to steatosis in mice fed the MCD diet, as this model is widely utilized to study mechanisms of NASH. Moreover, we systematically characterized the effect of MCP-1 deficiency on hepatocellular injury, hepatic inflammation, and fibrosis in mice fed the MCD diet.

\section{MATERIALS AND METHODS Mice}

All mice were used between 8 and 12 weeks of age. Male $\mathrm{MCP}^{-1}{ }^{-1-}$ mice $^{20}$ on a C57Bl/6J background and age-matched male $\mathrm{C} 57 \mathrm{Bl} / 6 \mathrm{~J}$ mice were purchased from The Jackson Laboratory (Bar Harbor, ME, USA). Mice were maintained in an AAALAC-accredited facility at the University of Kansas Medical Center. Mice were housed at an ambient temperature of $22^{\circ} \mathrm{C}$ with alternating 12-h light/dark cycles and supplied water and rodent chow (Teklad 8604; Harlan, Indianapolis, IN, USA) ad libitum before feeding custom diets. All animal procedures were performed according to the guidelines of the American Association for Laboratory Animal Science and were approved by the University of Kansas Medical Center Institutional Animal Care and Use Committee.

\section{Dietary Model of NASH}

The MCD rodent chow (MCD diet, 518810) and an otherwise identical diet sufficient in methionine and choline (control diet, 518754) were purchased from Dyets
(Bethlehem, PA, USA). Insofar as baseline differences in the purified diet, not just the methionine/choline deficiency, could affect baseline gene expression and other parameters, the use of this methionine/choline-sufficient diet is the most appropriate control. Mice were fed either control diet or MCD diet ad libitum for 4 weeks. The mice were not fasted before sample collection. Under isoflurane anesthesia, blood was then collected from the caudal vena cava into sodium citrate (final concentration, $0.38 \%$ ) for the collection of plasma and into an empty syringe for the collection of serum. Sections of liver from the left lateral lobe were fixed in $10 \%$ neutral-buffered formalin for $48 \mathrm{~h}$, and then embedded in paraffin. The right medial lobe was affixed to a cork with OCT (Fisher Scientific, Pittsburgh, PA, USA) and immersed for $3 \mathrm{~min}$ in liquid nitrogen-chilled isopentane. The remaining liver was snap frozen in liquid nitrogen.

\section{Histopathology, Sirius Red Staining, Clinical Chemistry, and Triglyceride Determination}

Formalin-fixed livers were sectioned at $5 \mu \mathrm{m}$, stained with hematoxylin and eosin ( $\mathrm{H}$ and $\mathrm{E}$ ), and evaluated by a pathologist (O.T.). Steatosis was scored from 0 to 4 in a masked fashion. Sirius red staining and fast-green counterstaining of formalin-fixed, paraffin-embedded liver sections were performed as described previously. ${ }^{21}$ The serum activity of alanine aminotransferase (ALT) was determined using a commercially available reagent (Thermo Fisher, Rockford, IL, USA). Hepatic triglyceride content was determined as described previously ${ }^{22}$ using commercially available reagents (Wako, Richmond, VA, USA). Serum triglyceride levels were determined using commercially available reagents (Pointe Scientific, Canton, MI, USA). Plasma glucose levels were determined using a commercial glucometer.

\section{Immunohistochemistry and Immunofluorescence}

Immunohistochemical staining of macrophages in frozen liver sections was performed as described ${ }^{11}$ utilizing a 1:1 mix of rat anti-mouse CD68/rat anti-mouse F4/80 antibodies (AbD Serotec, Raleigh, NC, USA) and Vector NovaRed substrate (Vector Laboratories, Burlingame, CA, USA). The extent of macrophage accumulation in liver sections stained immunohistochemically for macrophages was determined in a masked fashion, and each liver sample assigned a score from 1 to 10 . Oil Red O staining was performed as previously described. ${ }^{22}$ Immunofluorescent staining for type 1 collagen was performed as described previously ${ }^{23}$ utilizing a rabbit anti-mouse type 1 collagen antibody (Millipore, Billerica, MA, USA) and Alexa 594-conjugated secondary antibody (Invitrogen, Carlsbad, CA, USA). The slides were visualized using an Olympus BX41 microscope (Olympus, Lake Success, NY, USA). Images were captured using an Olympus DP70. Quantification of type 1 collagen antibody utilizing Scion Image software was performed as described previously. ${ }^{23}$ The evaluator was masked to both treatment and genotype. 


\section{Liver Homogenate Preparation and $\alpha$-Smooth Muscle Actin Western Blotting}

Whole liver homogenates were prepared by homogenizing $100 \mathrm{mg}$ of snap-frozen liver in $1 \mathrm{ml}$ of RIPA buffer containing protease inhibitor cocktail and PhosStop phosphatase inhibitor (Roche Applied Science). The homogenate was transferred to $1.7 \mathrm{ml}$ tubes, rotated end-over-end for $15 \mathrm{~min}$ at $4^{\circ} \mathrm{C}$, and then subjected to centrifugation at $14000 \mathrm{~g}$ for $10 \mathrm{~min}$. The supernatant was collected and protein concentration determined using the Bio-Rad DC protein assay (Bio-Rad, Hercules, CA, USA). A measure of $5 \mu \mathrm{g}$ of protein from each sample was diluted in $1 \times$ LDS buffer (Invitrogen, San Diego, CA, USA) and heated at $95^{\circ} \mathrm{C}$ in the presence of 2-mercaptoethanol for $10 \mathrm{~min}$. Samples were separated by electrophoresis utilizing precast 4-20\% Bis-tris criterion gels (Bio-Rad) and transferred to Immobilon PVDF membrane (Millipore) by semi-dry transfer. Membranes were blocked with $3 \%$ BSA in $1 \times$ TBST for $60 \mathrm{~min}$ at room temperature and then incubated overnight at $4{ }^{\circ} \mathrm{C}$ with a $1: 1000$ dilution of a monoclonal mouse anti$\alpha$-smooth muscle actin ( $\alpha$-SMA) antibody or 1:5000 dilution of a monoclonal mouse anti-glyceraldehyde 3-phosphate dehydrogenase (GAPDH) antibody (Millipore), followed by incubation for $1 \mathrm{~h}$ at room temperature with a secondary anti-mouse IgG-HRP-conjugated antibody diluted at 1:5000 (Cell Signaling Technology, Danvers, MA, USA). All antibodies were diluted in $1 \%$ BSA in $1 \times$ TBST. Membranes were then washed and incubated with Supersignal West Pico substrate (ThermoScientific) solution and exposed to Blue lite autoradiography film (ISC Bioexpress, Kaysville, UT, USA).

\section{RNA Isolation, CDNA Synthesis, and Real-Time PCR}

Total RNA was isolated from $\sim 100 \mathrm{mg}$ of snap-frozen liver using TRI reagent (Molecular Research Center, Cincinnati, OH, USA). A measure of $1 \mu \mathrm{g}$ of RNA was utilized for the synthesis of cDNA using a High-Capacity cDNA Reverse Transcription kit (Applied Biosystems, Foster City, CA, USA) and MyCycler thermal cycler (Bio-Rad). Levels of tumor necrosis factor $\alpha$ $(\mathrm{TNF} \alpha)$, cyclooxygenase-2 (COX-2), MCP-1, macrophage inflammatory protein (MIP-2, Cxcl2), keratinocyte chemoattractant/growth related gene (KC/Gro, Cxcl1), intercellular adhesion molecule-1 (ICAM-1), tissue inhibitor of metalloproteinase-1 (TIMP-1), connective tissue growth factor (CTGF), pro-collagen la1 (Colla1), transforming growth factor- $\beta$ (TGF- $\beta$ ), cell surface glycoprotein F4/80, macrosialin (CD68), interleukin (IL)- $1 \beta$, IL- 6 , and GAPDH mRNA were determined using the TaqMan gene expression assay from Applied Biosystems and TaqMan gene expression master mix (Applied Biosystems) on an ABI Prism 7300 sequence detection system (Applied Biosystems). The expression of each gene was adjusted relative to GAPDH expression levels, and relative expression level determined using the comparative $C_{\mathrm{t}}$ method.

\section{Statistics}

Comparison of two groups was performed using Student's $t$-test. Comparison of three or more groups was performed using one-way analysis of variance followed by the StudentNewman-Keuls post-hoc test. The criterion for statistical significance was $P<0.05$.

\section{RESULTS \\ Effect of MCP-1 Deficiency on MCD Diet-Induced Hepatocellular Injury}

In agreement with previous studies, ${ }^{11,24}$ hepatic MCP-1 mRNA levels increased in wild-type (WT) mice fed the MCD diet (Figure 1a). Serum ALT activity increased in mice fed the MCD diet compared with mice fed the control diet. Surprisingly, MCP-1 deficiency did not significantly affect serum ALT activity, although a trend toward higher serum ALT activity was observed in MCP-1-deficient mice fed the MCD diet $\left(P=0.075\right.$ for comparison between WT and $\mathrm{MCP}-1^{-1-}$ mice fed the MCD diet) (Figure 1b). This result indicates that
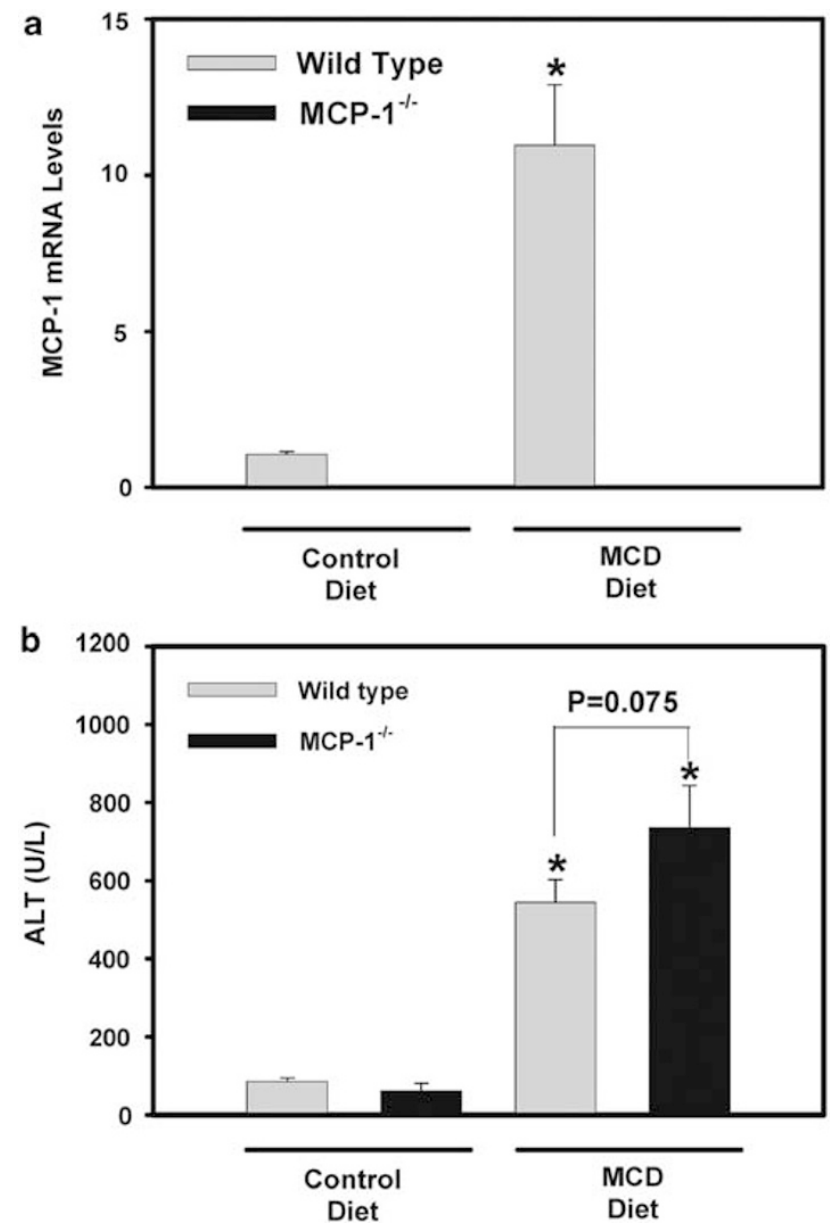

Figure 1 Effect of MCP-1 deficiency on MCP-1 mRNA levels and serum ALT activity in mice fed the MCD diet. MCP-1-deficient and wild-type mice, both on the $\mathrm{C} 57 \mathrm{BI} / 6 \mathrm{~J}$ genetic background, were fed either control diet or MCD diet for 28 days and (a) hepatic levels of MCP-1 mRNA and (b) serum ALT activity were determined. Data are expressed as mean \pm s.e.m. $N=4$ wild-type mice fed control diet, $3 \mathrm{MCP}-1^{-1-}$ mice fed control diet, 8 wildtype mice fed MCD diet, and $6 \mathrm{MCP}-1^{-1-}$ mice fed MCD diet. ${ }^{*}$, Significantly different from mice of the same genotype fed control diet. 

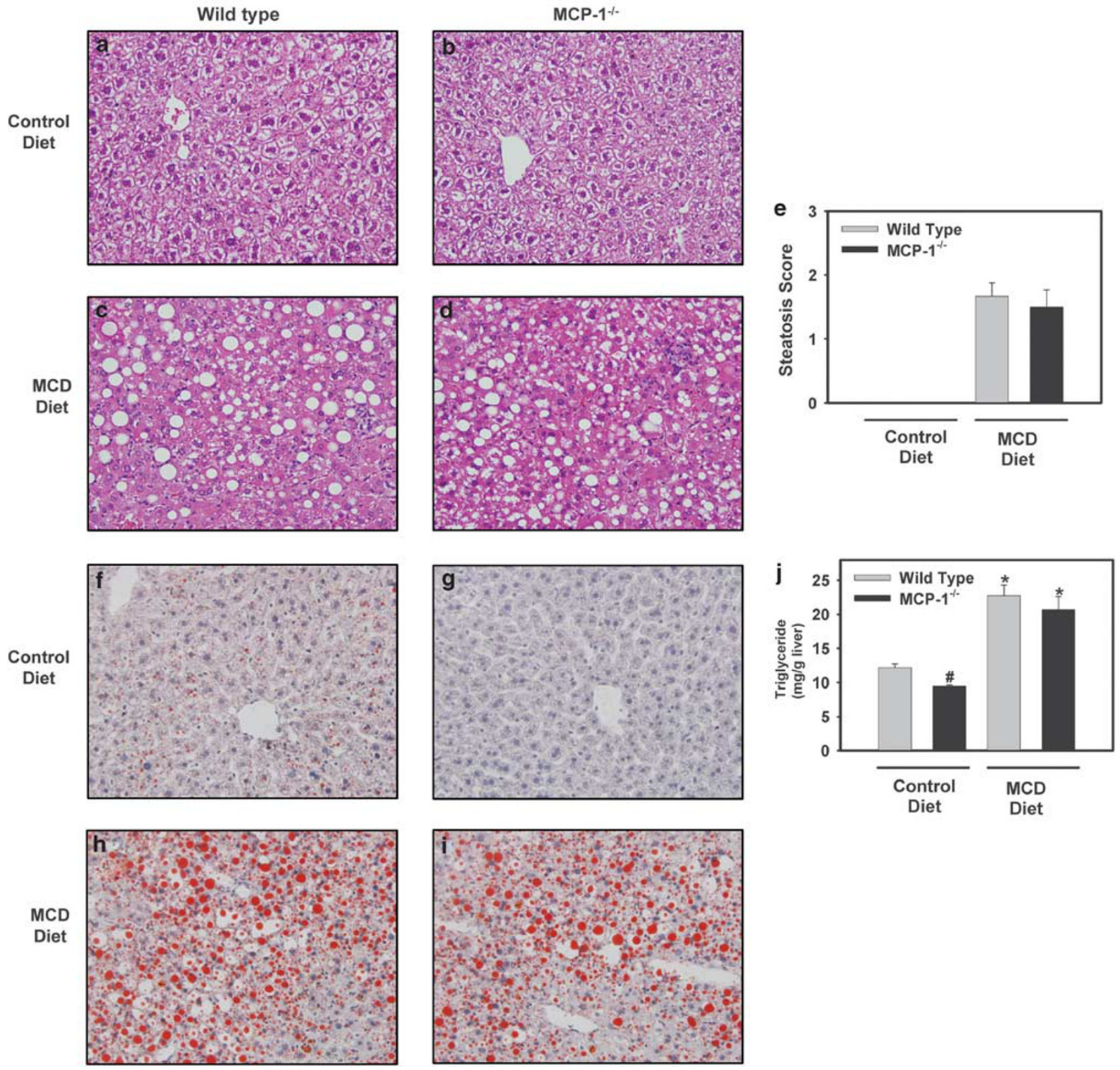

Figure 2 Effect of MCP-1 deficiency on liver histology and triglyceride levels in mice fed the MCD diet. MCP-1-deficient and wild-type mice, both on the $\mathrm{C} 57 \mathrm{BI} / 6 \mathrm{~J}$ genetic background, were fed either control diet or MCD diet for 28 days. Representative photomicrographs showing $\mathrm{H}$ and $\mathrm{E}$-stained liver sections (a-d) and Oil Red O-stained liver sections (f-i) from wild-type mice fed control diet (a, f) or MCD diet (c, h) and from MCP-1-deficient mice fed control diet $(\mathbf{b}, \mathbf{g})$ or MCD diet $(\mathbf{d}, \mathbf{i})$. (e) $\mathrm{H}$ and E-stained slides were evaluated by a pathologist and assigned a macrovesicular steatosis severity score. (j) Hepatic triglyceride levels were determined as described in Materials and Methods. Data are expressed as mean \pm s.e.m. $N=4$ wild-type mice fed control diet, $3 \mathrm{MCP}-1^{-/-}$mice fed control diet, 8 wild-type mice fed MCD diet, and $6 \mathrm{MCP}-1^{-1-}$ mice fed MCD diet. ${ }^{*}$, Significantly different from mice of the same genotype fed control diet. \#, Significantly different from wild-type mice fed the control diet.

MCP-1 deficiency does not reduce hepatocellular injury in mice fed the MCD diet.

\section{Effect of MCP-1 Deficiency on MCD Diet-Induced Steatosis}

The $\mathrm{H}$ and E-stained slides were examined by a pathologist and no substantial differences in liver histology were observed between WT mice and MCP-1-deficient mice fed control diet (Figure 2a and b). Both WT and MCP-1-deficient mice fed the MCD diet developed panacinar macrovesicular hepatic steatosis of similar severity (Figure $2 \mathrm{c}-\mathrm{e}, \mathrm{h}$ and $\mathrm{i}$ ). Hepatic triglyceride levels increased significantly in both WT and MCP-1-deficient mice fed the MCD diet (Figure 2j). These results are in contrast to mice fed a high-fat diet, in which both steatosis and hepatic triglyceride levels were reduced by MCP-1 deficiency. ${ }^{2,3}$ Although the mice were not 
fasted, changes in circulating triglyceride and glucose levels mirrored previous studies. ${ }^{25,26}$ Serum triglyceride levels were significantly reduced in mice fed the MCD diet (control vs MCD diet, mean \pm s.e.m.: WT mice, $57 \pm 6$ vs $24 \pm 1 \mathrm{mg}$ per $100 \mathrm{ml}$; $\mathrm{MCP}-1^{-I-}$ mice, $57 \pm 13$ vs $28 \pm 2 \mathrm{mg}$ per $100 \mathrm{ml}$ ). Similarly, plasma glucose levels were significantly reduced in mice fed the MCD diet (control vs MCD diet, mean \pm s.e.m.: WT mice, $289 \pm 43$ vs $160 \pm 11 \mathrm{mg}$ per $100 \mathrm{ml}$; $\mathrm{MCP}_{-1}-^{-1}$ mice, $348 \pm 34$ vs $146 \pm 23 \mathrm{mg}$ per $100 \mathrm{ml}$ ). Interestingly, glucose levels were higher in MCP-1 ${ }^{-1-}$ mice fed control diet compared with WT mice fed control diet.

Of importance, MCP-1 deficiency significantly reduced baseline triglyceride levels in mice fed control diet (Figure 2j). Similarly, Oil Red O staining indicated a decrease in lipid content of livers from MCP-1-deficient mice fed control diet (Figure 2f-g).

\section{Effect of MCP-1 Deficiency on MCD Diet-Induced Hepatic Inflammatory Gene Induction and Macrophage Accumulation}

Compared with WT mice fed control diet, levels of ICAM-1, MIP-2, and KC/Gro $\alpha$ mRNA were increased in livers of MCP1-deficient mice fed control diet (Figure $3 \mathrm{a}-\mathrm{c}$ ). Previous studies have identified a similar, and likely compensatory, effect of MCP-1 deficiency on the expression of other chemokines. ${ }^{27}$ Interestingly, IL-1 $\beta$ mRNA levels were lower in MCP- $1^{-1-}$ mice fed the MCD diet, although this did not achieve statistical significance (Figure 3d). Induction of ICAM-1, MIP-2, KC/Gro, IL-1 $\beta$, COX-2, and TNF $\alpha$ mRNA in livers of mice fed the MCD diet was unaffected by MCP-1 deficiency (Figure 3a-f). Hepatic IL-6 mRNA levels were not significantly affected by either the MCD diet or MCP-1 deficiency (Figure 3g).

Scattered sinusoidal macrophage staining was observed in the livers of WT and MCP-1-deficient mice fed control diet (Figure $4 \mathrm{a}$ and b), representing Kupffer cells. Hepatic macrophage accumulation increased in mice fed the MCD diet and was not affected by MCP-1 deficiency (Figure 4c-e). In agreement with this observation, liver CD68 and F4/80 mRNA levels tended to increase in mice fed the MCD diet, and were unaffected by MCP-1 deficiency (Figure $4 \mathrm{f}$ and $\mathrm{g}$ ).

\section{Effect of MCP-1 Deficiency on Profibrogenic Gene Expression and Collagen Deposition in Livers of Mice Fed the MCD Diet}

MCP-1 has been implicated in hepatic fibrosis and has been shown to directly activate stellate cells. ${ }^{15,19}$ This suggests that MCP- 1 could affect liver fibrosis without altering steatosis or inflammation. Therefore, we also determined the effect of MCP-1 deficiency on induction of various profibrogenic genes. Induction of Colla1, CTGF, and TGF- $\beta$ mRNA, but not TIMP-1 mRNA, were significantly reduced in MCP-1deficient mice fed the MCD diet (Figure 5a-d). Moreover, hepatic collagen deposition, determined utilizing immunofluorescent staining for type 1 collagen (Figure $6 \mathrm{a}-\mathrm{d}$ ) and sirius red staining (6f-i), was decreased in MCP-1-deficient mice fed the MCD diet. Quantification of the type 1 collagen staining indicated a significant reduction in collagen protein levels in the livers of MCP-1-deficient mice fed the MCD diet (Figure 6e). The levels of $\alpha$-SMA increased significantly in mice fed the MCD diet, and this was reduced in MCP-1deficient mice (Figure 6j). Densitometry indicated a significant reduction in $\alpha$-SMA protein in MCP-1-deficient mice fed the MCD diet compared with WT mice fed the MCD diet (mean \pm s.e.m., $n=6,1.5 \pm 0.25$ (WT) vs $0.8 \pm 0.1$ $\left.\left(\mathrm{MCP}^{-1}{ }^{-l-}\right)\right)$.

\section{DISCUSSION}

The MCD diet is widely utilized to evaluate mechanisms of NASH in rodents. However, key differences in the mechanism of hepatic steatosis among the multiple animal models of NASH make translation of the results to human disease challenging. We found that MCP-1 did not contribute to macrovesicular steatosis in mice fed the MCD diet, in striking contrast to models of NAFLD/NASH induced by feeding a high-fat diet. ${ }^{2,3,12}$ Indeed, triglyceride accumulation in livers of mice fed the MCD diet is mediated through multiple mechanisms, including an altered balance in fatty acid uptake and secretion of triglycerides, increased de novo lipogenesis, and increased triglyceride synthesis. ${ }^{5,28}$ High levels of sucrose in the diet contribute to the increased lipogenesis and triglyceride synthesis, but not the abnormal fatty acid uptake and triglyceride secretion. ${ }^{5}$ Indeed, simple replacement of sucrose with starch in the MCD diet formulation largely prevents hepatic steatosis in this model, with only minimal effect on loss of body weight. ${ }^{5}$ The complex mechanisms of severe hepatic steatosis induced by the MCD diet may bypass the requirement of MCP-1 to induce steatosis. Interestingly, MCP-1 deficiency significantly reduced hepatic triglyceride levels and Oil Red O staining in livers of mice fed methionine/choline-sufficient control diet, which is high in sucrose such as the MCD diet. This rather subtle change in baseline hepatic lipid levels in mice fed the control diet may reflect the defined role of MCP-1 in hepatic lipid accumulation, glucose regulation, and insulin resistance induced by diets high in sugar. $^{2,29}$

In models of Western diet-induced NAFLD, the role of MCP-1 in liver inflammation is potentially secondary to its role in promoting steatosis, although several studies suggest potential disconnects between lipid accumulation and liver inflammation in NAFLD. For example, the two diacylglycerol acyltransferases (DGATs) have different effects on the pathogenesis of NASH in mice fed the MCD diet. Inhibition of DGAT1 has no effect on triglycerides or inflammation but decreases fibrosis, ${ }^{30}$ whereas inhibition of DGAT2 decreases triglyceride synthesis and steatosis but increases inflammation and fibrosis. ${ }^{31}$ In mice fed a diet high in sucrose or fat, there was an increase in both steatosis and inflammation compared with control mice. ${ }^{32}$ However, human consumption of fructose correlated with decreased steatosis, and 

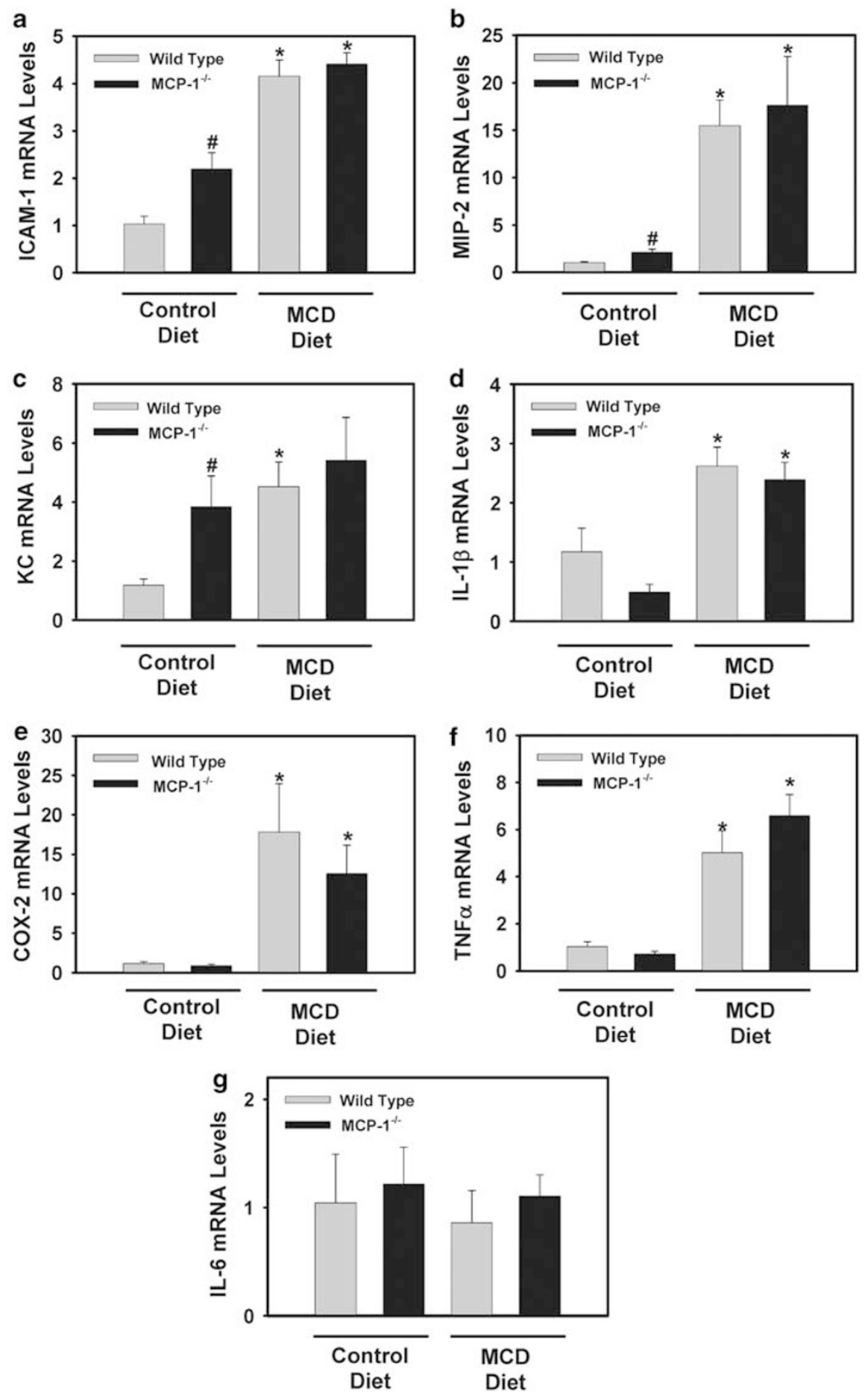

Figure 3 Effect of MCP-1 deficiency on hepatic proinflammatory gene mRNA levels in mice fed the MCD diet. MCP-1-deficient and wild-type mice, both on the $\mathrm{C} 57 \mathrm{BI} / 6 \mathrm{~J}$ genetic background, were fed either control diet or MCD diet for 28 days. Hepatic levels of (a) ICAM-1, (b) MIP-2, (c) KC/Gro $\alpha$, (d) IL-1 $\beta$, (e) COX-2, (f) TNF $\alpha$, and (g) IL-6 mRNAs were quantified. Data are expressed as mean \pm s.e.m. $N=4$ wild-type mice fed control diet, $3 \mathrm{MCP}-1^{-/-}$mice fed control diet, 8 wild-type mice fed MCD diet, and $6 \mathrm{MCP}-1^{-1-}$ mice fed MCD diet. *, Significantly different from mice of the same genotype fed control diet. ", Significantly different from wild-type mice fed the control diet.

increased inflammation and fibrosis. ${ }^{33}$ The lack of effect of MCP-1 deficiency on the pathogenesis of steatosis and inflammation in mice fed the MCD diet is somewhat unexpected when compared with the effect of MCP-1 deficiency on liver pathology in mice fed a high-fat diet. However, the mechanisms of liver inflammation in mice fed 

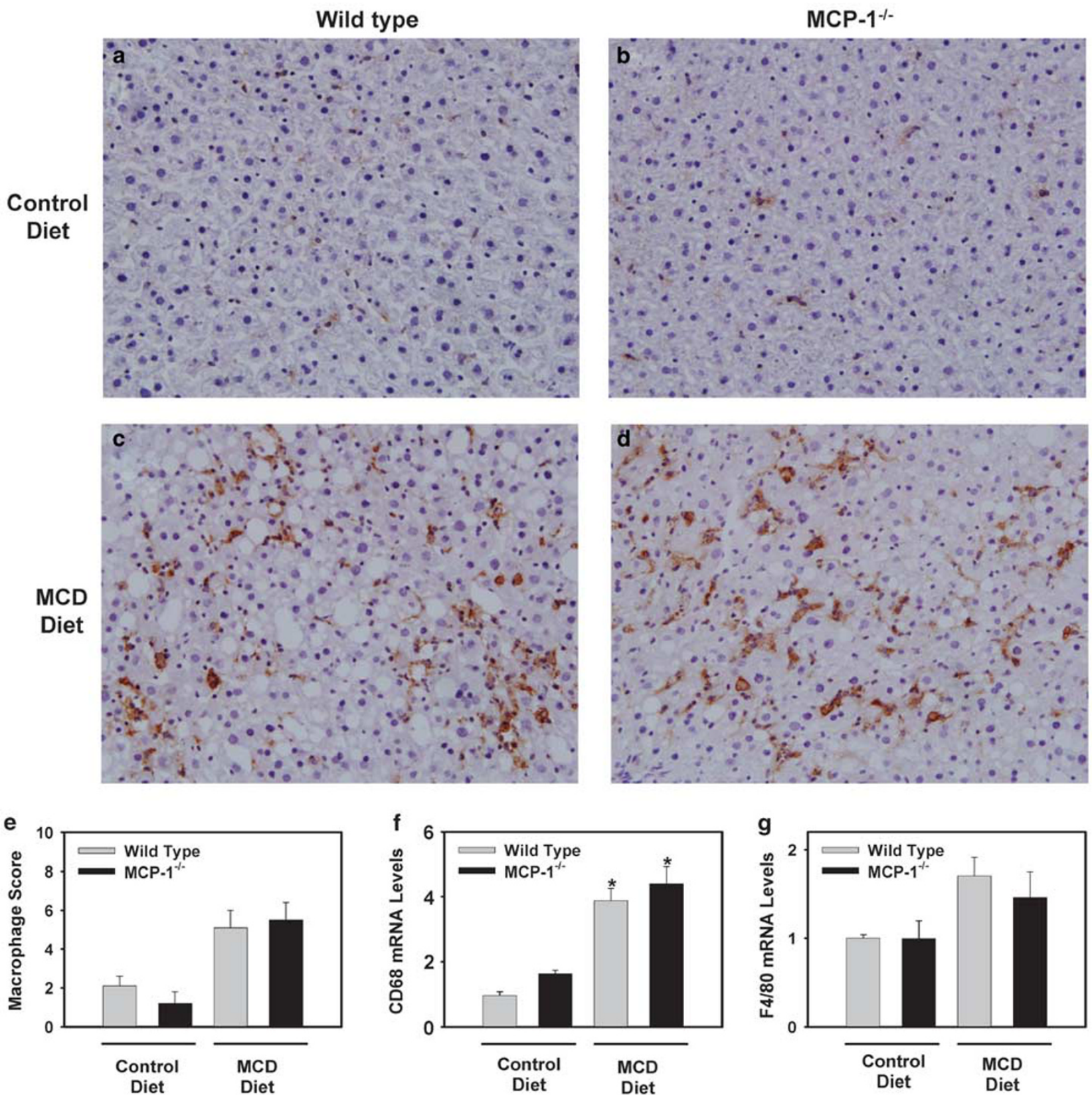

Figure 4 Effect of MCP-1 deficiency on hepatic macrophage accumulation. MCP-1-deficient and wild-type mice, both on the C57BI/6J genetic background, were fed either control diet or MCD diet for 28 days. Representative photomicrographs show combined CD68- and F4/80-positive staining (ie, macrophages) in livers of wild-type mice fed control diet (a) or MCD diet (c) and from MCP-1-deficient mice fed control diet (b) or MCD diet (d). (e) The extent of macrophage accumulation was assessed in a masked fashion and each tissue assigned a score (1-10). Hepatic levels of (f) CD68 and (g) F4/80 mRNA were determined. $N=4$ wild-type mice fed control diet, $3 \mathrm{MCP}-1^{-/-}$mice fed control diet, 8 wild-type mice fed MCD diet, and $6 \mathrm{MCP}-1^{-/-}$mice fed $\mathrm{MCD}$ diet. *, Significantly different from mice of the same genotype fed control diet.

the MCD diet are complex and deletion of a single chemokine may not have profound effects.

Indeed, intracellular signaling pathways such as the JNK $\mathrm{MAPK},{ }^{34}$ transcription factors such as $\mathrm{CEBP} / \beta^{35}$ and NF$\kappa \mathrm{B},{ }^{24,36}$ and nuclear receptors such as $\mathrm{FXR}^{37}$ and PPARs ${ }^{38,39}$ contribute to hepatic expression of numerous proinflammatory mediators including ICAM-1, cytokines such as TNFa, IL-6, and MCP-1, as well as osteopontin and COX-2. ${ }^{24,25,35,40}$ As many of these mediators contribute to the pathogenesis of steatohepatitis in the MCD diet model, their expression was evaluated in the present study. Of importance, the exact role of some cytokines, such as TNF $\alpha$ and IL-6, remains unclear. More recently, we showed that coagulation protease signaling through protease-activated receptor-1 

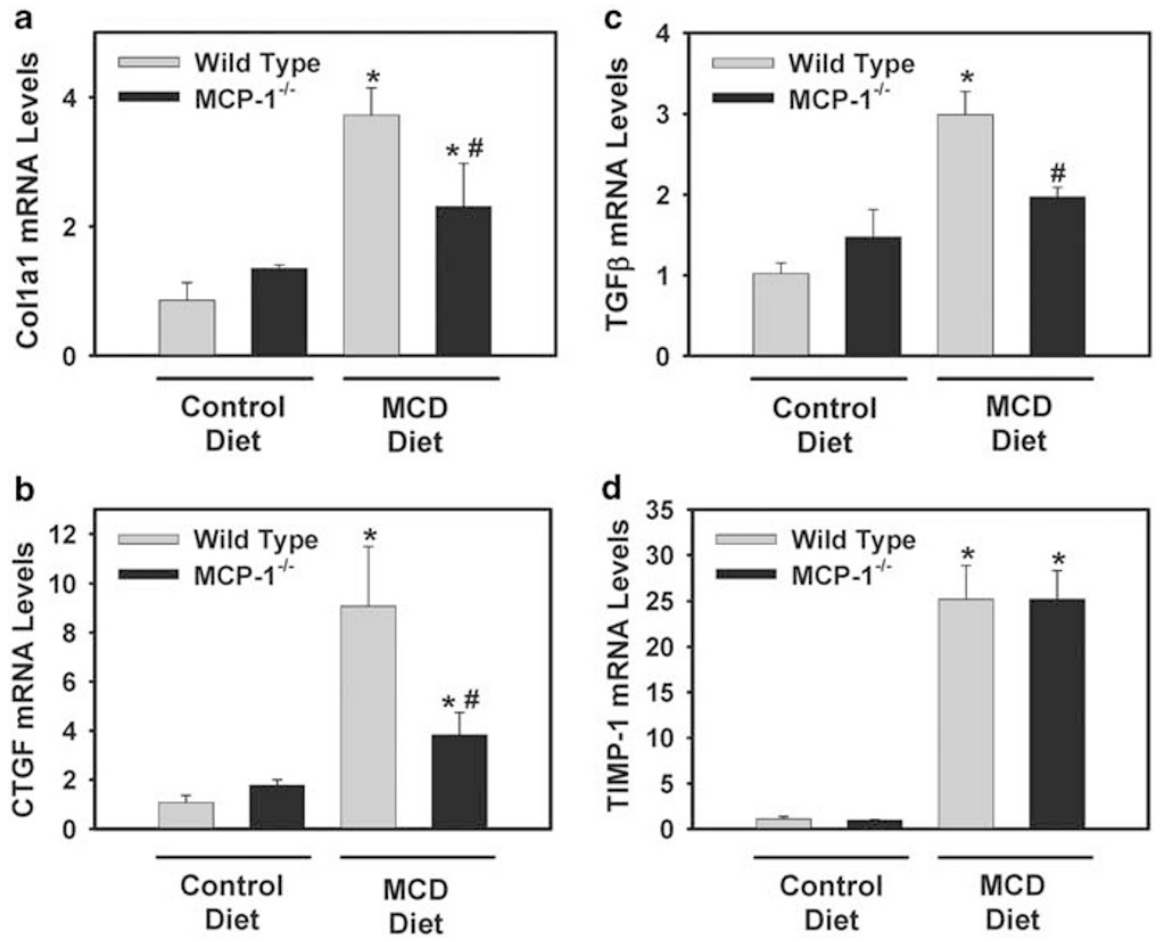

Figure 5 Effect of MCP-1 deficiency on levels of profibrogenic genes in the livers of mice fed the MCD diet. MCP-1-deficient and wild-type mice, both on the C57BI/6J genetic background, were fed either control diet or MCD diet for 28 days. Hepatic levels of (a) Col1a1, (b) CTGF, (c) TGF $\beta$, and (d) TIMP-1 were quantified. Data are expressed as mean \pm s.e.m. $N=4$ wild-type mice fed control diet, $3 \mathrm{MCP}-1^{-1-}$ mice fed control diet, 8 wild-type mice fed MCD diet, and $6 \mathrm{MCP}-1^{-1-}$ mice fed MCD diet. ${ }^{*}$, Significantly different from mice of the same genotype fed control diet. ", Significantly different from wild-type mice fed the MCD diet.

(PAR-1) is a central amplifier of MCP-1 induction and the inflammatory response in livers of mice fed the MCD diet. ${ }^{11}$

Although several of these studies have clarified the mechanism whereby MCP-1 expression increases in mice fed the MCD diet, the results presented here indicate that MCP- 1 is not required for the full manifestation of hepatic inflammation in mice fed the MCD diet. This is in contrast to other models of liver injury in which interventions targeting MCP-1 or its primary receptor CCR2 significantly reduce tissue macrophage accumulation. ${ }^{12,15}$ The spectrum of inflammatory mediators induced in livers of mice fed the MCD diet is broad, and these other mediators may be sufficient to induce liver inflammation. Moreover, compensatory induction of other chemokines in MCP-1-deficient mice has been observed previously, ${ }^{27}$ and could account for the lack of effect of MCP-1 deficiency on hepatic inflammation in mice fed the MCD diet. Of importance, MCP-1 clearly contributes to the pathogenesis of NAFLD in other models, and mechanisms of increased MCP-1 expression in other models may be similar to the MCD diet.

The mechanism of liver fibrosis in the MCD diet model is largely considered to stem from the chronic inflammatory response elicited by steatosis and hepatocellular injury. Indeed, numerous studies indicate that interventions reducing steatosis also reduce inflammation and fibrosis. ${ }^{41,42}$ It is initially counterintuitive that profibrogenic gene expression and collagen expression were reduced in $\mathrm{MCP}-1^{-1-}$ mice fed the MCD diet, with no effect on steatosis or inflammation. However, mediators produced secondary to steatosis could contribute to more distal processes in MCD diet-induced steatohepatitis without impacting steatosis. For example, we have shown that PAR-1 deficiency reduces MCP-1 expression, but not steatosis, in mice fed the MCD diet. ${ }^{11}$ Moreover, other interventions have been shown to reduce fibrosis without impact on steatohepatitis in mice fed the MCD diet. ${ }^{43}$ Our results indicate that the profibrogenic effects of MCP-1 in this model of steatohepatitis are independent of inflammation. However, the expression of TIMP-1, an inhibitor of metalloproteinases, was unaffected by MCP-1 deficiency. Of importance, the expression of TIMP-1 in this model is driven by TNF, which was also unaffected by MCP-1 deficiency. ${ }^{44}$

Multiple studies indicate that MCP-1 activates cultured hepatic stellate cells and portal fibroblasts, ${ }^{15,45-47}$ and stellate cells are the principal cell type producing collagen in NASH. ${ }^{15,48}$ Interestingly, we found that hepatic levels of $\alpha$-SMA, an indicator of stellate cell activation, were reduced in livers of $\mathrm{MCP}-1^{-/-}$mice fed the MCD diet.

In summary, MCP-1 deficiency did not impact liver steatosis or inflammation in mice fed the MCD diet. However, MCP-1 deficiency reduced profibrogenic gene expression and liver fibrosis in this model. Of interest, MCP-1 deficiency 

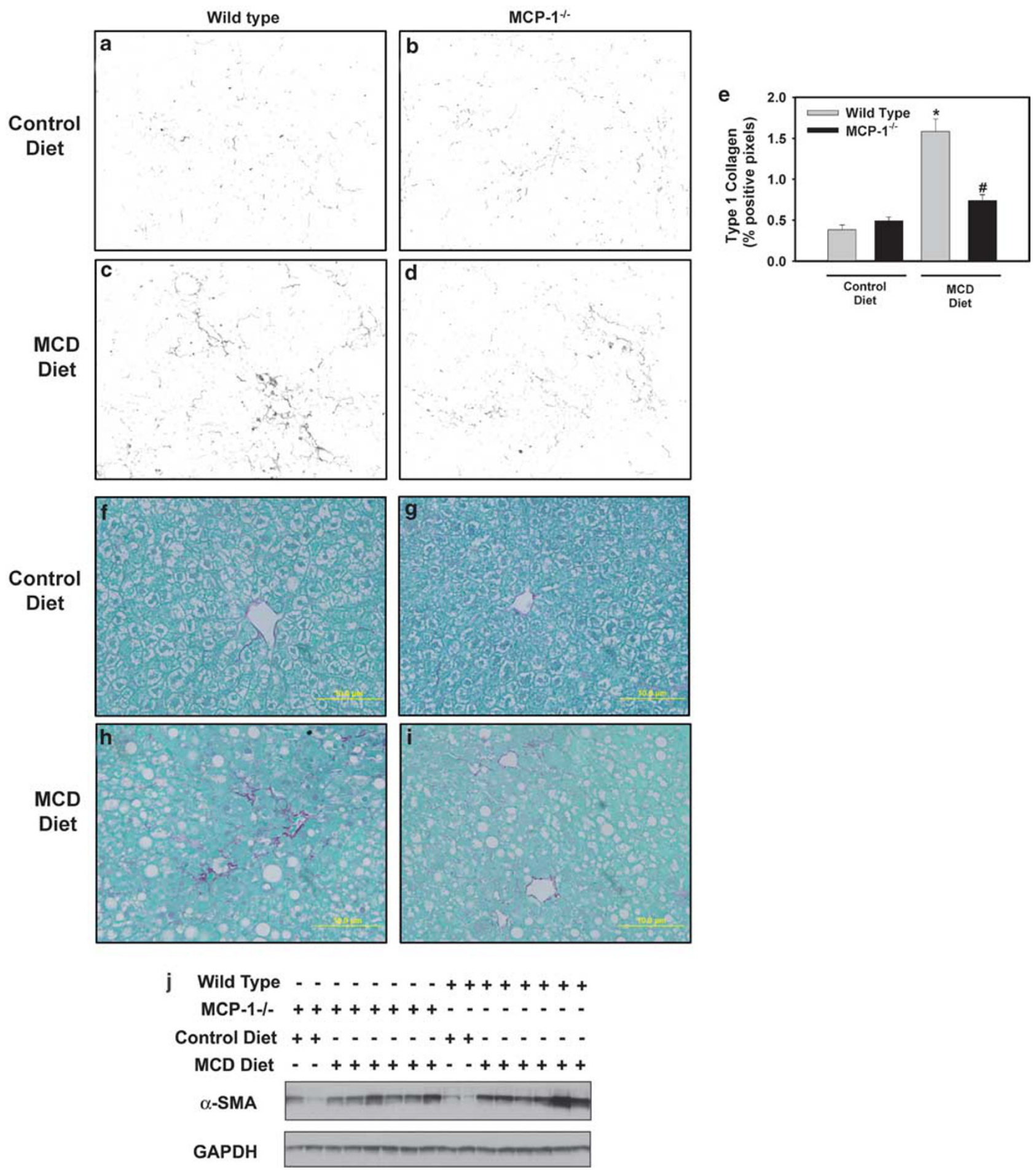

Figure 6 Effect of MCP-1 deficiency on collagen and $\alpha$-SMA protein levels in livers of mice fed the MCD diet. MCP-1-deficient and wild-type mice, both on the $\mathrm{C} 57 \mathrm{BI} / 6 \mathrm{~J}$ genetic background, were fed either control diet or MCD diet for 28 days. Representative photomicrographs showing liver sections stained for (a-d) type 1 collagen (black) and (f-i) sirius red-stained liver sections from wild-type mice fed control diet $(\mathbf{a}, \mathbf{f})$ or $\mathrm{MCD}$ diet $(\mathbf{c}, \mathbf{h})$, and from MCP-1-deficient mice fed control diet $(\mathbf{b}, \mathbf{g})$ or MCD diet $(\mathbf{d}, \mathbf{i})$. (e) Quantification of positive staining for type 1 collagen was performed using morphometry as described in Materials and Methods. Data are expressed as mean \pm s.e.m. (j) Western blot showing $\alpha$-SMA protein levels in liver. $N=4$ wild-type mice fed control diet, $3 \mathrm{MCP}-1^{-1-}$ mice fed control diet, 8 wild-type mice fed MCD diet, and $6 \mathrm{MCP}-1^{-1-}$ mice fed MCD diet. ${ }^{*}$, Significantly different from mice of the same genotype fed control diet. ", Significantly different from wild-type mice fed the MCD diet. 
selectively reduced the expression of several fibrogenic genes and collagen deposition in mice fed the MCD diet without impacting steatohepatitis. This is likely the result of direct effects of MCP-1 on stellate cell activation. ${ }^{15}$ This result highlights an important distinction in the mechanism of steatosis in the MCD diet model of NASH compared with other models. The MCD diet model may represent a novel opportunity to investigate profibrogenic effects of MCP-1 outside the scope of its role in steatosis or inflammation.

\section{ACKNOWLEDGEMENTS}

This study was supported by the American Heart Association Scientist Development Grant $0835121 \mathrm{G}(\mathrm{JPL})$ and by the National Institutes of Health P20 RR021940 (JPL) and R01DK081343 (GLG). We thank Huina Cai, Ruipeng (Frank) Wang and Noriko Esterly for outstanding technical assistance.

\section{DISCLOSURE/CONFLICT OF INTEREST}

The authors declare no conflict of interest.

1. Anstee QM, Goldin RD. Mouse models in non-alcoholic fatty liver disease and steatohepatitis research. Int J Exp Pathol 2006;87:1-16.

2. Kanda H, Tateya S, Tamori Y, et al. MCP-1 contributes to macrophage infiltration into adipose tissue, insulin resistance, and hepatic steatosis in obesity. J Clin Invest 2006;116:1494-1505.

3. Rull A, Rodriguez F, Aragones G, et al. Hepatic monocyte chemoattractant protein-1 is upregulated by dietary cholesterol and contributes to liver steatosis. Cytokine 2009;48:273-279.

4. Tous M, Ferre N, Rull A, et al. Dietary cholesterol and differential monocyte chemoattractant protein-1 gene expression in aorta and liver of apo E-deficient mice. Biochem Biophys Res Commun 2006;340:1078-1084.

5. Pickens MK, Yan JS, Ng RK, et al. Dietary sucrose is essential to the development of liver injury in the MCD model of steatohepatitis. J Lipid Res 2009;50:2072-2082.

6. Rinella ME, Green RM. The methionine-choline deficient dietary model of steatohepatitis does not exhibit insulin resistance. J Hepatol 2004:40:47-51.

7. Haukeland JW, Damas JK, Konopski Z, et al. Systemic inflammation in nonalcoholic fatty liver disease is characterized by elevated levels of CCL2. J Hepatol 2006;44:1167-1174.

8. Westerbacka J, Kolak M, Kiviluoto T, et al. Genes involved in fatty acid partitioning and binding, lipolysis, monocyte/macrophage recruitment, and inflammation are overexpressed in the human fatty liver of insulin-resistant subjects. Diabetes 2007;56:2759-2765.

9. Marsillach J, Oliveras-Ferraros C, Beltran R, et al. Serum concentrations of extracellular fatty acid synthase in patients with steatohepatitis. Clin Chem Lab Med 2009;47:1097-1099.

10. Greco D, Kotronen A, Westerbacka J, et al. Gene expression in human NAFLD. Am J Physiol Gastrointest Liver Physiol 2008;294:G1281-G1287.

11. Luyendyk JP, Sullivan BP, Guo GL, et al. Tissue factor deficiency and protease activated receptor-1 deficiency reduce inflammation elicited by diet-induced steatohepatitis in mice. Am J Pathol 2010;176:177-186.

12. Tamura $Y$, Sugimoto $M$, Murayama $T$, et al. Inhibition of CCR2 ameliorates insulin resistance and hepatic steatosis in $\mathrm{db} / \mathrm{db}$ mice. Arterioscler Thromb Vasc Biol 2008;28:2195-2201.

13. Deshmane SL, Kremlev S, Amini $\mathrm{S}$, et al. Monocyte chemoattractant protein-1 (MCP-1): an overview. J Interferon Cytokine Res 2009;29: 313-326.

14. Dambach DM, Watson LM, Gray KR, et al. Role of CCR2 in macrophage migration into the liver during acetaminophen-induced hepatotoxicity in the mouse. Hepatology 2002;35:1093-1103.

15. Seki E, De MS, Inokuchi S, et al. CCR2 promotes hepatic fibrosis in mice. Hepatology 2009;50:185-197.

16. Kuziel WA, Morgan SJ, Dawson TC, et al. Severe reduction in leukocyte adhesion and monocyte extravasation in mice deficient in CC chemokine receptor 2 (gene targeting/monocyte chemoattractant protein 1/trafficking/peritonitis/granuloma). Proc Natl Acad Sci USA 1997;94:12053-12058.
17. Zamara E, Galastri S, Aleffi S, et al. Prevention of severe toxic liver injury and oxidative stress in MCP-1-deficient mice. J Hepatol 2007;46:230-238.

18. Ramm GA, Shepherd RW, Hoskins AC, et al. Fibrogenesis in pediatric cholestatic liver disease: role of taurocholate and hepatocyte-derived monocyte chemotaxis protein-1 in hepatic stellate cell recruitment. Hepatology 2009;49:533-544.

19. Wynn TA. Cellular and molecular mechanisms of fibrosis. J Pathol 2008;214:199-210.

20. Lu B, Rutledge BJ, Gu L, et al. Abnormalities in monocyte recruitment and cytokine expression in monocyte chemoattractant protein 1-deficient mice. J Exp Med 1998;187:601-608.

21. Bergheim I, Guo L, Davis MA, et al. Critical role of plasminogen activator inhibitor-1 in cholestatic liver injury and fibrosis. J Pharmacol Exp Ther 2006;316:592-600.

22. Tanaka Y, Aleksunes LM, Yeager RL, et al. NF-E2-related factor 2 inhibits lipid accumulation and oxidative stress in mice fed a high-fat diet. J Pharmacol Exp Ther 2008;325:655-664.

23. Kim ND, Moon JO, Slitt AL, et al. Early growth response factor-1 is critical for cholestatic liver injury. Toxicol Sci 2006;90:586-595.

24. Yu J, Ip E, Dela PA, et al. COX-2 induction in mice with experimental nutritional steatohepatitis: role as pro-inflammatory mediator. Hepatology 2006;43:826-836

25. Mas E, Danjoux M, Garcia V, et al. IL-6 deficiency attenuates murine diet-induced non-alcoholic steatohepatitis. PLoS One 2009;4:e7929.

26. Koppe SW, Sahai A, Malladi P, et al. Pentoxifylline attenuates steatohepatitis induced by the methionine choline deficient diet. J Hepatol 2004;41:592-598.

27. Ferreira AM, Rollins BJ, Faunce DE, et al. The effect of MCP-1 depletion on chemokine and chemokine-related gene expression: evidence for a complex network in acute inflammation. Cytokine 2005;30:64-71.

28. Rinella ME, Elias MS, Smolak RR, et al. Mechanisms of hepatic steatosis in mice fed a lipogenic methionine choline-deficient diet. J Lipid Res 2008;49:1068-1076.

29. Tamura Y, Sugimoto M, Murayama T, et al. C-C chemokine receptor 2 inhibitor improves diet-induced development of insulin resistance and hepatic steatosis in mice. J Atheroscler Thromb 2010;17:219-228.

30. Yamaguchi K, Yang L, McCall S, et al. Diacylglycerol acyltranferase 1 anti-sense oligonucleotides reduce hepatic fibrosis in mice with nonalcoholic steatohepatitis. Hepatology 2008;47:625-635.

31. Yamaguchi K, Yang L, McCall S, et al. Inhibiting triglyceride synthesis improves hepatic steatosis but exacerbates liver damage and fibrosis in obese mice with nonalcoholic steatohepatitis. Hepatology 2007;45: 1366-1374.

32. Li Z, Soloski MJ, Diehl AM. Dietary factors alter hepatic innate immune system in mice with nonalcoholic fatty liver disease. Hepatology 2005;42:880-885.

33. Abdelmalek MF, Suzuki A, Guy C, et al. Increased fructose consumption is associated with fibrosis severity in patients with nonalcoholic fatty liver disease. Hepatology 2010;51:1961-1971.

34. Schattenberg JM, Singh R, Wang Y, et al. JNK1 but not JNK2 promotes the development of steatohepatitis in mice. Hepatology 2006;43: $163-172$.

35. Rahman SM, Schroeder-Gloeckler JM, Janssen RC, et al. CCAAT/ enhancing binding protein beta deletion in mice attenuates inflammation, endoplasmic reticulum stress, and lipid accumulation in diet-induced nonalcoholic steatohepatitis. Hepatology 2007;45: 1108-1117.

36. Dela PA, Leclercq I, Field J, et al. NF-kappaB activation, rather than TNF, mediates hepatic inflammation in a murine dietary model of steatohepatitis. Gastroenterology 2005;129:1663-1674.

37. Zhang $S$, Wang J, Liu $Q$, et al. Farnesoid $X$ receptor agonist WAY362450 attenuates liver inflammation and fibrosis in murine model of non-alcoholic steatohepatitis. J Hepatol 2009;51:380-388.

38. Ip E, Farrell GC, Robertson G, et al. Central role of PPARalphadependent hepatic lipid turnover in dietary steatohepatitis in mice. Hepatology 2003;38:123-132.

39. Leclercq IA, Lebrun VA, Starkel $P$, et al. Intrahepatic insulin resistance in a murine model of steatohepatitis: effect of PPARgamma agonist pioglitazone. Lab Invest 2007;87:56-65.

40. Sahai $A$, Malladi $P$, Melin-Aldana $H$, et al. Upregulation of osteopontin expression is involved in the development of nonalcoholic steatohepatitis in a dietary murine model. Am J Physiol Gastrointest Liver Physiol 2004;287:G264-G273. 
41. Titos E, Ferre N, Lozano JJ, et al. Protection from hepatic lipid accumulation and inflammation by genetic ablation of 5-lipoxygenase. Prostaglandins Other Lipid Mediat 2010;92:54-61.

42. Miura K, Kodama Y, Inokuchi S, et al. Toll-like receptor 9 promotes steatohepatitis via induction of interleukin-1beta in mice. Gastroenterology 2010;139:323-334.

43. Velayudham A, Dolganiuc A, Ellis $M$, et al. VSL\#3 probiotic treatment attenuates fibrosis without changes in steatohepatitis in a diet induced nonalcoholic steatohepatitis model in mice. Hepatology 2008;49:989-997.

44. Tomita K, Tamiya G, Ando S, et al. Tumour necrosis factor alpha signalling through activation of Kupffer cells plays an essential role in liver fibrosis of non-alcoholic steatohepatitis in mice. Gut 2006;55:415-424.
45. Marra F, Romanelli RG, Giannini C, et al. Monocyte chemotactic protein-1 as a chemoattractant for human hepatic stellate cells. Hepatology 1999;29:140-148.

46. Kruglov EA, Nathanson RA, Nguyen T, et al. Secretion of MCP-1/CCL2 by bile duct epithelia induces myofibroblastic transdifferentiation of portal fibroblasts. Am J Physiol Gastrointest Liver Physiol 2006;290: G765-G771.

47. Sprenger $H$, Kaufmann A, Garn $H$, et al. Differential expression of monocyte chemotactic protein-1 (MCP-1) in transforming rat hepatic stellate cells. J Hepatol 1999;30:88-94.

48. Washington $\mathrm{K}$, Wright $\mathrm{K}$, Shyr $\mathrm{Y}$, et al. Hepatic stellate cell activation in nonalcoholic steatohepatitis and fatty liver. Hum Pathol 2000;31: 822-828. 